\title{
Promoter selectivity of the Bacillus subtilis response regulator DegU, a positive regulator of the fla/che operon and sacB Kensuke Tsukahara and Mitsuo Ogura*
}

\author{
Address: Institute of Oceanic Research and Development, Tokai University, 3-20-1 Orido-Shimizu, Shizuoka 424-8610, Japan \\ Email: Kensuke Tsukahara - astronotus_ocellatus@docomo.ne.jp; Mitsuo Ogura* - oguram@scc.u-tokai.ac.jp \\ * Corresponding author
}

Published: 15 January 2008

BMC Microbiology 2008, 8:8 doi:| 0.| | 86/|47|-2/80-8-8

This article is available from: http://www.biomedcentral.com/I47|-2/80/8/8

(C) 2008 Tsukahara and Ogura; licensee BioMed Central Ltd.

This is an Open Access article distributed under the terms of the Creative Commons Attribution License (http://creativecommons.org/licenses/by/2.0), which permits unrestricted use, distribution, and reproduction in any medium, provided the original work is properly cited.
Received: 2I August 2007

Accepted: 15 January 2008

\begin{abstract}
Background: The response regulator DegU and its cognate histidine kinase DegS constitute a two-component system in the Gram-positive soil bacterium Bacillus subtilis. Unphosphorylated and phosphorylated forms of DegU are known to activate target gene transcription in B. subtilis. Although phosphorylated DegU (DegU-P) regulates more than one hundred and twenty genes, the targets of unphosphorylated DegU are unknown, except for comK.
\end{abstract}

Results: We found that the fla/che (flagella and chemotaxis) operon is positively regulated by unphosphorylated DegU. The effect was most prominent in a strain bearing the functional swrAA gene, a positive regulator of fla/che. Unphosphorylated DegU bound to two regions in the fla/che regulatory region containing an inverted repeat-like sequence that resembles the inverted repeat (IR) in the comK promoter. Mutational analysis revealed that positive regulation of fla/che by SwrAA requires DegU-binding. An analysis of the DegU-P-regulated gene sacB (levansucrase gene) by footprint and mutational analyses revealed that DegU-P bound to a direct repeat (DR) of the DegU-recognition motifs, which has been shown to be functional in vivo, while unphosphorylated DegU did not. These results strongly suggest that the arrangement of the DegU-binding motifs determines whether unphosphorylated DegU or DegU-P binds to the sacB promoter. The hypothesis was confirmed by observing degS-independent expression when the DR in the sacB-lacZ fusion was changed to an IR, suggesting that unphosphorylated DegU regulates the sacB promoter through the newly created IR. This was confirmed by binding of unphosphorylated DegU to the IR in the sacB promoter.

Conclusion: This study demonstrated that $\mathrm{DegU}$ positively regulates $f g B$ and $\operatorname{sac} B$ through its binding to the promoter regions. We demonstrated that DegU-P prefers binding to DR but not to IR in the sacB promoter.

\section{Background}

To respond to environmental fluctuations, bacteria employ a large and elaborate family of two-component signaling systems. The classical two-component system consists of a sensor kinase and its cognate response regulator [1]. In response to the signal input, the kinase phos- phorylates its own histidine residue. The phosphoryl group is then transferred to a conserved aspartate residue on the cognate response regulator, which then acts as a transcription factor in most cases. Given the many studies on how response regulator regulates output response by phosphorylation, it is not surprising that variable strate- 
gies were found [1]. Upon phosphorylation, some regulators dimerize to be activated or interact with other proteins or DNA [2], while other regulators are relieved from inhibition by their N-terminal domain [3]. One such two-component system in the Gram-positive soil bacterium Bacillus subtilis consists of the response regulator DegU and its cognate histidine kinase DegS. DegU belongs to the NarL family, whose members have a helixturn-helix structure at their C-terminus [4]. The DegSDegU system regulates many cellular processes, including exoprotease production and competence development [49]. It has also been reported to sense salt stress and to mediate appropriate responses $[7,10,11]$. In addition, it was found recently that the protein machinery for chromosome separation (SMC-ScpA-ScpB) forms a complex with DegS and inhibits its kinase activity [12]. The activity of DegU itself has been shown to be finely tuned by several factors. The Rap-Phr systems are regulatory machinery to receive extracellular signals [13]. DegU is negatively regulated by RapG since the protein inhibits its DNAbinding activity [14]. RapG activity is in turn inhibited by its cognate extracellular pentapeptide PhrG after the peptide is taken up by the cell. Thus, the RapG-PhrG system functions as a positive regulatory mechanism for DegU. Moreover, the transcription of rapG is repressed by RghR [15].

Unphosphorylated DegU is required for competence development and binds to the promoter region of $\operatorname{comK}$, which encodes a master regulator of competence development $[16,17]$. Unphosphorylated DegU has also been reported to facilitate the binding of ComK to the $\operatorname{comK}$ promoter [18]. Previously we identified a DegU-recognized incomplete inverted repeat (IR) on the comK promoter (GTCATTTA-N7-TAAATATC) by using various mutated comK-lacZ fusions [19]. Additional targets of unphosphorylated DegU have not been identified.

Phosphorylated DegU (DegU-P) activates the expression of more than one hundred twenty genes, including $a \mathrm{prE}$ (which encodes alkaline protease) and $s a c B$ (which encodes levansucrase); it also represses wapA, which encodes a cell-wall associated protein $[6-9,20]$. In addition, the expression of $b p r$, which encodes bacillopeptidase $\mathrm{F}$, has been reported to be probably dependent on DegU-P $[8,21]$. To date, the DNA recognition sequence of DegU-P has not been identified with the exception of $a \mathrm{prE}$ and $b p r$. Our analysis revealed that an important cis-factor for DegU-dependent aprE expression is a direct repeat (DR) of the downstream half of the DegU-recognized IR in the $\operatorname{comK}$ promoter with two-nucleotide spacing (-70 to -52 relative to the transcription start site), [19]. In addition, we identified three DRs with zero or two-nucleotide spacing, which are important for DegU-binding to the $b p r$ promoter region and DegU-P-dependent expression of $b p r$
[22]. Furthermore, overproduction of DegU or the degU32 mutation, which renders DegU-P resistant to dephoshorylation, resulted in a decrease in the expression of the fla/ che operon encoding chemotaxis-related proteins and components of the flagella apparatus $[5,8,9,23,24]$.

Since DegU-P stimulates the transcription of many genes and unphosphorylated DegU is required for $\operatorname{comK}$ transcription, DegU is regarded as a molecular switch that controls cell fate [5]. However, what factor determines promoter selectivity of DegU-P and unphosphorylated DegU remains unclear.

In this paper, we found that $f \lg B$, which is the first gene of the 26-kb-long fla/che operon, is subject to direct positive regulation by unphosphorylated DegU through two DegU-binding sequences containing an IR-like sequence. In contrast, footprint analyses of the $s a c B$ promoter revealed that DegU-P bound to a DR within the DegUbinding sequence. Since unphosphorylated DegU bound to $f \lg B$, the arrangement of the DegU-binding sequence within promoter regions (DR or IR-like sequence) must dictate which form of DegU (phosphorylated or unphosphorylated) binds to them. This hypothesis was confirmed by expression analysis of sacB-lacZ fusions carrying an artificial IR within their DegU-binding sequence.

\section{Results}

\section{Unphosphorylated DegU binds to two IR-like sequences in flgB}

While studying the interaction of DegU with its target promoters, we found that unphosphorylated DegU bound to two DegU-binding regions present within flgB (BR1 and BR2). BR1 seems to contain an IR-like sequence (the -95 to -69 region relative to the transcription start site) with long spacing (eleven bases), and BR2 also seems to contain an IR-like sequence (the +119 to +136 region relative to the transcription start site) with two-nucleotide spacing (Figure 1). Next, we performed footprint analysis using His-tagged DegU and obtained essentially similar protection profiles to those obtained by using untagged DegU (Figure 2). Previously, gel retardation analysis using unphosphorylated His-tagged DegU showed that DegU bound to an upstream region from the transcription start site of $f \lg B$, although the binding sequence was not identified [24]. It should be noted that each of the half sites of these two IR-like sequences shows similarity to the DegUrecognition sequences in the $\operatorname{comK}$ promoter, although they are not perfect IR sequences. Furthermore, phosphorylation of DegU by His-tagged DegS abrogated its binding to BR1 but not that to BR2. Based on these results, we concluded that unphosphorylated DegU binds to two regions containing the IR-like sequence in the $\mathrm{flgB}$ regulatory region. 
A

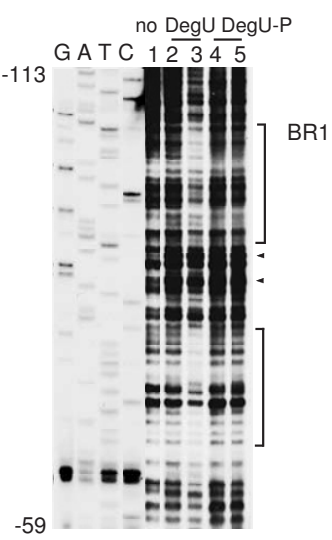

no DegU DegU-P

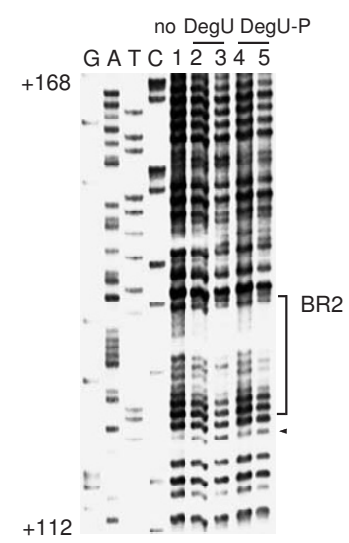

B

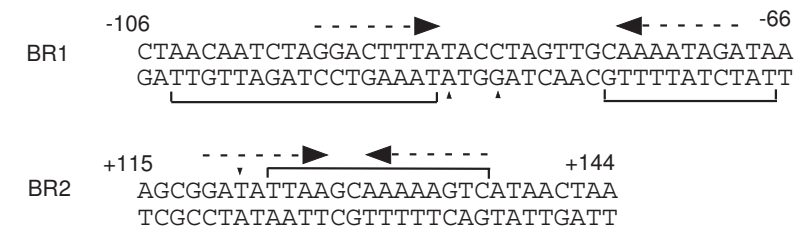

Figure I

Footprint analysis of the flgB promoter. A. The probes were prepared by PCR amplification. To analyze the upstream ( -120 to +38 , the bottom strand in the left panel) and downstream $(+15$ to +264 , the top strand in the right panel) regions, the oligonucleotide pairs flgB-F and flgB-D-bio, and flgB-DU-bio and flgB-DD, respectively, were used. The sequencing ladder templates for the upstream and downstream region analysis were generated by PCR using the oligonucleotide pairs flgB-F and flgB-D, and flgB-DU and flgB-DD, respectively. The probes $(40 \mathrm{nM})$ were incubated with increasing amounts of DegU and His-DegS in the presence or absence of ATP, and subjected to DNase I cleavage. The sequencing ladder is shown in lanes G, A, T and C. The brackets and arrowheads indicate the protected regions and hypersensitive sites, respectively. I. No protein. 2 and $4,0.15 \mu \mathrm{M}$ of DegU and $0.05 \mu \mathrm{M}$ His-tagged DegS; 3 and $5,0.3 \mu \mathrm{M}$ of DegU and 0.I $\mu$ M His-tagged DegS. 2 and 3, no ATP; 4 and 5, I mM ATP. B. The nucleotide sequences of the regulatory regions are shown. The numbers indicate the nucleotide positions relative to the transcription start site. Dotted arrows above the sequences indicate the putative DegUbinding motifs.

\section{DegU and SwrAA regulation of the expression of a FID fusion carrying two $B R s$}

To examine the in vivo functions of the BRs, we constructed a F1D fusion carrying both BRs and measured the $\beta$-galactosidase activities of the fusion in various genetic backgrounds, including disruption mutation of degS or $\operatorname{deg} U$ in the presence of active or inactive swrAA (Figure 3 ). The degS mutation is an in-frame deletion, which results in acting as a non-polar type mutation to the downstream degU gene. The swrAA gene has been reported to enhance flgB expression by an unknown mechanism [25]. In addition, this gene carries a frame-shift mutation in the 168 strain [26]. The transcription of the flgB fusion is solely driven by sigma A type RNA polymerase, as the fusion lacks a promoter for sigma D type RNA polymerase [23]. In the strain bearing functional swrAA at an ectopic thrC locus, the expression of F1D was about four-fold higher than that in the 168 (swrAA) strain, which is consistent
A
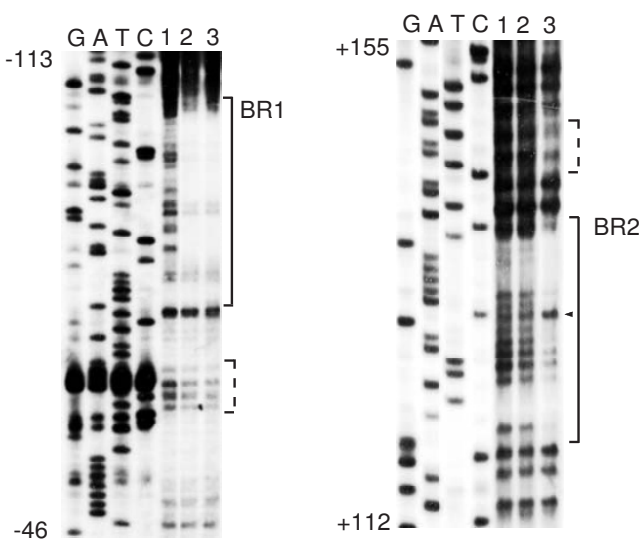

B

BR1

$-106$ CTAACAATCTAGGACTTTATACCTAGTTGCAAAATAGATAA GATTGTTAGATCCTGAAATATGGATCAACGTTTTATCTATT

BR2

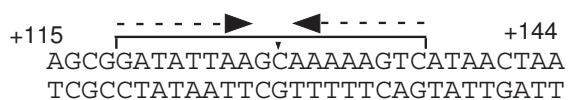

\section{Figure 2}

Footprint analysis of the flgB promoter using His-DegU. A. Preparation of the probes and sequencing ladders was the same as that in Figure I. For footprint assays, we used the procedures developed for biotinylated DNA probes as described previously [47]. The sequencing ladder is shown in lanes G, A, T and C. The brackets, dotted brackets and arrowheads indicate the protected regions, weakly protected regions and hypersensitive sites, respectively. I. No protein. 2, $0.15 \mu \mathrm{M}$ of His-DegU; 3, $0.3 \mu \mathrm{M}$ of His-DegU. No His-tagged DegS was added. B. The nucleotide sequences of the promoter region are shown. Dotted arrows above the sequences indicate the putative DegU-binding motifs. The numbers indicate the nucleotide positions relative to the transcription start site.

with former results [25]. As unphosphorylated DegU binds to two BRs, it was expected that unphosphorylated DegU would regulate the expression of F1D. If this were the case, then only the degU mutation but not the degS mutation would have an effect on fusion expression. As shown in Figure $3 \mathrm{~A}$, in swrAA and swrA $A^{+}$backgrounds, the deg $U$ mutation decreased the expression of F1D, while the degS mutation had little or no effect. The effect of the $\operatorname{deg} U$ mutation was more prominent in the swrA $A^{+}$background than in the swrAA background. These results indicated that unphosphorylated DegU positively regulates F1D fusion expression. Moreover, the degU32 mutation, which makes DegU-P resistant to dephosphorylation [5], reduced the expression of F1D 3-fold and 1.7-fold in the swrAA and swrAA+ backgrounds, respectively. This observation is in good agreement with former results [8,9] (Figure $3 \mathrm{~B})$.

\section{DegU and SwrAA act on flgB expression through BRI}

To determine the in vivo role of BR1 in the positive regulation of $f \lg B$ by $D e g U$, we constructed a fusion carrying 
A

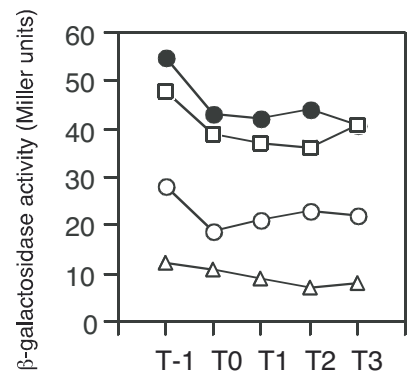

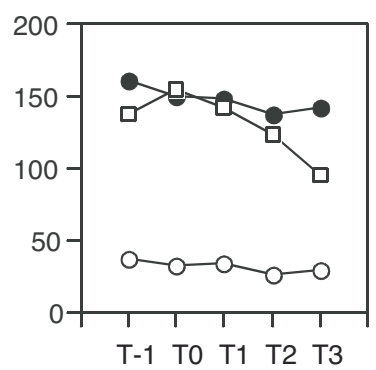

C

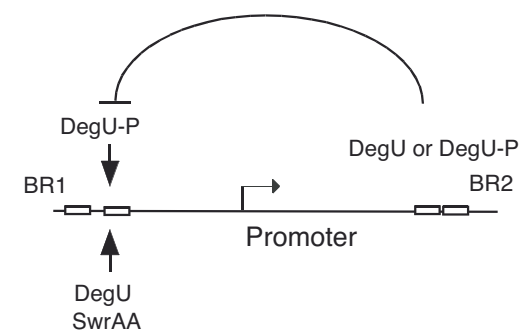

B

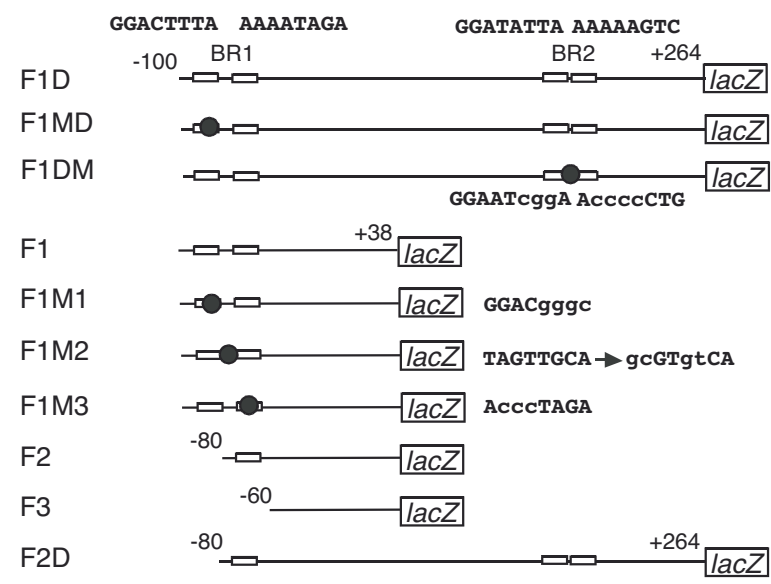

swrAA

\begin{tabular}{|c|c|c|c|c|c|c|c|}
\hline WT & $\operatorname{degs}$ & \multicolumn{2}{|c|}{$\operatorname{deg} U$ degU32 } & WT & $\operatorname{deg} S$ & \multicolumn{2}{|c|}{$\operatorname{deg} U$ degU32 } \\
\hline 51 & 48 & 31 & 17 & 191 & 202 & 47 & 120 \\
\hline 34 & 45 & 38 & 16 & 38 & 35 & 32 & 31 \\
\hline 50 & 44 & 32 & 143 & 154 & 148 & 28 & 198 \\
\hline 61 & 60 & 29 & 160 & 282 & 222 & 44 & 279 \\
\hline 39 & 38 & 54 & 40 & 58 & ND & ND & ND \\
\hline 30 & 38 & 37 & 36 & 44 & ND & ND & ND \\
\hline 32 & 26 & 29 & 19 & 20 & ND & ND & ND \\
\hline 38 & 37 & 36 & 55 & 37 & 49 & 32 & ND \\
\hline 26 & 22 & 21 & 35 & 39 & ND & ND & ND \\
\hline 22 & 19 & 18 & 27 & 23 & 33 & 27 & 15 \\
\hline
\end{tabular}

Figure 3

DegU-regulation of flgB. Cells were grown in MC medium. Their $\beta$-galactosidase activities were determined as described previously [45]. A. Left (swrAA) and right $\left(s w r A A^{+}\right)$panels show representative results of the $\beta$-galactosidase assays. All strains carry the flgB-lacZ (FID) fusion. Filled circles, wild type (OAM 385 in the left and OAM386 in the right); squares, degS (OAM388 in the left and OAM390 in the right); open circles, degU (OAM387 in the left and OAM389 in the right); triangles, degU32, (OAM39I in the left). The numbers on the $x$ axis represent the growth time in hours relative to the end of vegetative growth (TO). B. Transcription of various flgB-lacZ fusions in each genetic background. The open rectangles and filled circles on the line indicate the DegU-binding motifs and introduced mutations, respectively. The mutant fusions tested included the FI to F3 series of fusions (the $3^{\prime}$ ends are +38 ) as well as FID and F2D (the $3^{\prime}$ ends are +264), in which increasing deletions were made from the $5^{\prime}$ end, and the $M$ series, in which point mutations (small letters indicate replaced nucleotides) were introduced. FIMD and FIMI contain the same mutation. All fusions were constructed by cloning various PCR products into pIS284. Consequently, all strains are derivatives of OAM385 (amyE::flgB-lacZ). The oligonucleotides used to construct the pIS284 derivatives are shown in Additional file 1 . The activities were assayed hourly from T-I through to T3. At least three independent assays were performed and the averages of the peak values are shown. Standard deviations did not exceed $20 \%$. The "degS", "degU" and "degU32" columns show the $\beta$-galactosidase activities of each fusion in the degS::Emr, degU:: $\mathrm{Km}^{r}$ and degU32 backgrounds, respectively. ND means no data. C. Schematic representation of the regulation of flgB. Arrows and a curved T-bar indicate activation and inhibition, respectively.

BR1 alone (F1) and tested its expression in various genetic backgrounds (Figure 3B). As well as F1D, the expression of F1 was decreased in the degU but not degS background, suggesting positive regulation of F1 by unphosphorylated DegU. The reduced expression in the presence of the degU mutation and the increased expression in the presence of functional swrAA were abolished by introducing point mutations into the upstream and downstream half sites of the IR-like sequences in BR1 and the long spacing region (F1M1, F1M3 and F1M2, respectively) or by deleting BR1 (F2 and F3). These results demonstrated that unphosphorylated DegU binding to BR1 is required for the positive regulation of $f l g B$ by DegU, and for the enhancing effect of SwrAA. This result strongly suggested that at least part of the positive function of SwrAA is exercised through DegU.
In fact, in the $\operatorname{deg} U$ strain carrying F1D, F1DM or F1, SwrAA had little effect on the expression of $f l g B$. Surprisingly, the introduction of the degU32 mutation into a strain carrying F1 and swrAA resulted in about a three-fold increase in the expression of F1, which is not consistent with the results of the expression analysis of F1D. This positive effect of degU32 in the swrAA strain was abolished by disrupting or deleting BR1, suggesting that higher cellular concentrations of DegU-P may positively regulate $f l g B$ expression through increased binding of DegU-P to BR1. However, this hypothesis is not in agreement with the result shown in Figure 1. We will discuss this discrepancy with respect to the binding of DegU to BR1 (Discussion). 


\section{$B R 2$ appears to prevent the positive regulation of flgB by} DegU-P through BRI

To investigate the in vivo role of $\mathrm{BR} 2$, we constructed a F2D fusion carrying an incomplete BR1, BR2, and the core promoter, and tested its $\beta$-galactosidase activities in various genetic backgrounds. The levels of expression of F2D in all of the genetic backgrounds tested were similar to those of the F3 fusion, which should reflect strength of the core promoter of flgB. Moreover, the absence of effects of $\operatorname{deg} U$ and functional swrAA in F1MD was reasonable, because F1MD is equivalent to F2D with respect to distribution of BRs. These results suggested that BR2 is not involved in positive regulation by SwrAA and unphosphorylated DegU. A comparison of the expression patterns of the F1D and F1DM fusions showed that there was essentially no difference except for the epistatic effect of the degU32 mutation. This suggested that BR2 could have some role to play in the negative effect of the degU32 mutation on F1D expression. In the presence of BR2, the degU32 mutation did not exhibit positive regulation of flgB through BR1, while, in the absence of BR2, the mutation resulted in increased fusion expression. Thus, BR2 appears to prevent the positive regulation of $f l g B$ by DegU$\mathrm{P}$ through BR1, leading to downregulation of F1D in degU32 cells (Figure 3C). In addition, DegU-P bound to BR2 could not serve as a repressor, because there was no significant effect of the degU32 mutation on F2D expression. It should be noted that the slight reducing effect of the degU32 mutation on F1MD was observed in the swrAA background. This is due to an unknown reason.

\section{Transcription start site of sacB}

To characterize the interaction of DegU-P with its targets, we selected $s a c B$, which is a well-characterized gene belonging to the DegU-P regulon. The DegU-P dependency of $s a c B$ expression has been reported previously [27]. We determined the transcription start site of $s a c B$ by primer extension analysis (data not shown). A previous analysis found that the transcription start site is sevenbase downstream of the newly determined site [28]. This discrepancy is due to an unknown reason. The numbering of nucleotide of $s a c B$ was done based on our result hereafter.

\section{Footprint analysis of the sacB promoter region}

To further characterize the interaction of DegU with the $s a c B$ promoter, we performed DNase I footprint analysis (Figure 4). Unphosphorylated DegU had no effect on DNase I cleavage of the $s a c B$ promoter region, while DegU-P protected the several regions on the both strands from DNase I cleavage. These results are consistent with DegU-P-dependent regulation of the $s a c B$ gene observed in vivo. The region containing a DR (-105 to -90) of the putative DegU-binding sequence was only protected on the both strands. In addition, we found that His-tagged

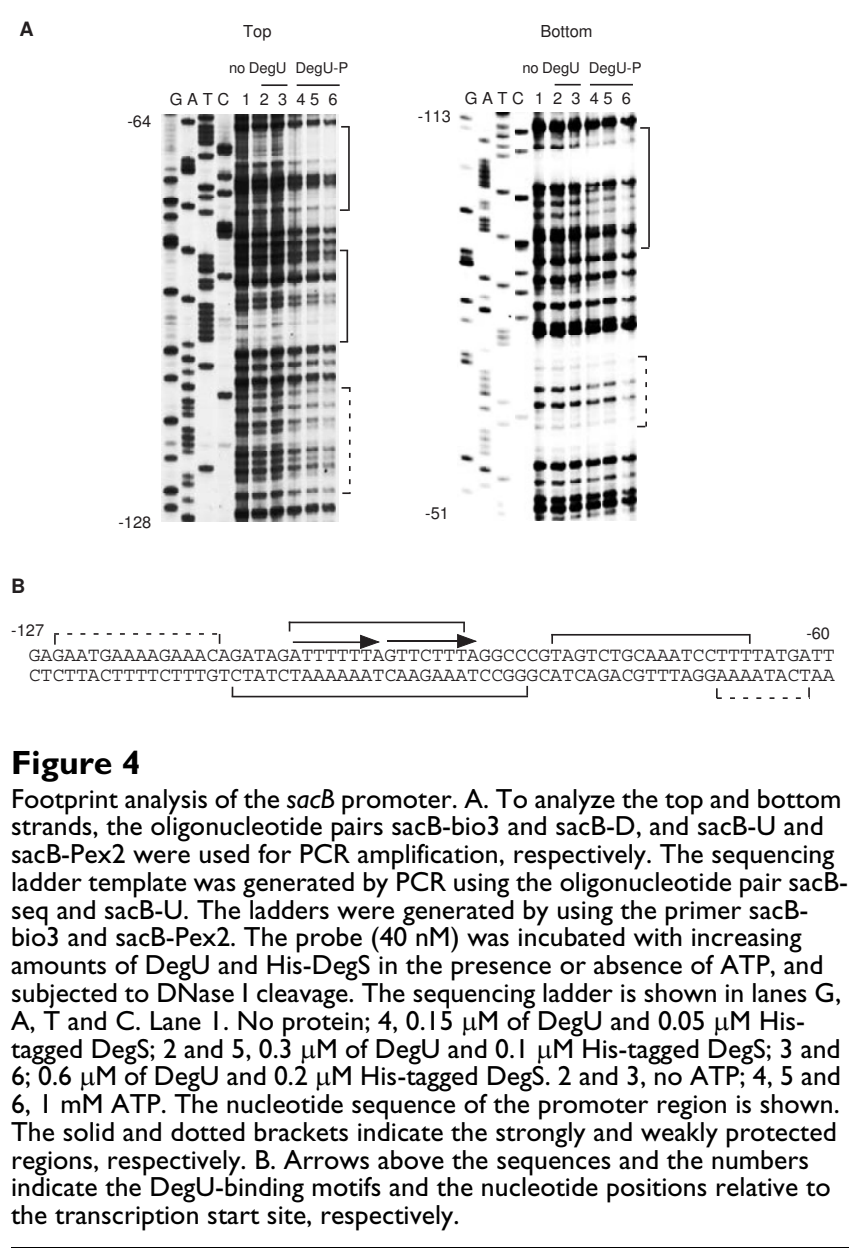

unphosphorylated DegU was able to bind to the $s a c B$ promoter region in footprint analysis for an unknown reason (see Discussion). In the experiments using His-tagged DegU the region containing the DR was protected from DNase I cleavage (Figure 5).

\section{Confirmation of the in vivo function of the detected $D R$ by lacZ fusion analysis}

To confirm the functions of the detected DegU-binding regions in vivo, we performed lac $Z$ fusion analysis. The expression of $s a c B$ is known to be regulated in two ways, namely, by DegU-P and by the antitermination system involving SacY. It has been shown previously that the upstream and downstream regions of the core promoter are required for the former and latter regulatory mechanisms, respectively, and that the deletion of the downstream region resulted in high constitutive expression of $s a c B$ in the absence of sucrose [27]. Thus, we only fused the upstream region of $s a c B$ to the lac $Z$ gene. Sequential deletion of this $s a c B-l a c Z$ fusion revealed the importance of the -117 to -97 region in the $s a c B$ promoter (Figure 6). We introduced various point mutations and a deletion into the -115 to -60 region, which contains the DegU-P 
A

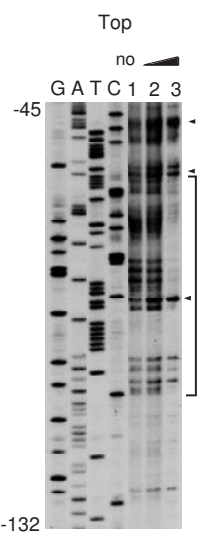

B

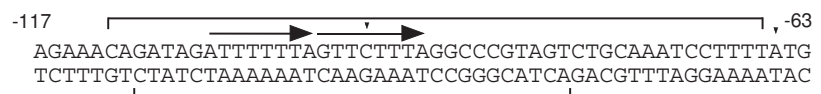

Figure 5

Footprint analysis of the $s a c B$ promoter by using His-DegU. A. Preparation of the probes and the sequencing ladders was the same as that in figure 4 . The probes $(40 \mathrm{nM})$ were incubated with increasing amounts of His-tagged DegU $(0.3$ and $0.6 \mu \mathrm{M})$ and subjected to $\mathrm{DNase}$ I cleavage. For footprint assays, we used the procedures developed for biotinylated DNA probes as described previously [47]. The sequencing ladder is shown in lanes G, A, T and $C$. The nucleotide sequence of the promoter region is shown. The brackets and arrowheads indicate the protected regions and hypersensitive sites, respectively. B. Arrows above the sequence indicate the DegUbinding motifs. The numbers indicate the nucleotide positions relative to the transcription start site.

binding sites, and examined their $\beta$-galactosidase activities. Especially, the deletion of the region containing the DR resulted in the strongest effect on $s a c B$ expression (Sdel4) among the mutants tested. In addition, the M1 and M2 mutations disrupting the DR led to significant decreases in sacB-lacZ expression. These results confirmed the functionality of the DR. Furthermore the other detected DegU-binding sites did not have significant in vivo roles, because the introduced mutations to the regions showed marginal effects on transcription of the fusions. We note that the mutation located immediately downstream of the DR (S-M12) reduced fusion expression to some extent. Notably, a previous deletion study had shown that the ability of the degU32 mutation to enhance $s a c B$ expression is dependent on the -137 to -90 region of the $s a c B$ promoter [29]; this region contains the DR we identified. Another interesting point is that the nucleotide change that yields the S-M3 point mutant makes the sequence of the upstream motif closer to the DegU-Pbinding consensus motif (Figure 6B); this led to a 2.5 -fold higher level of expression of the fusion compared to that of S-WT.
A

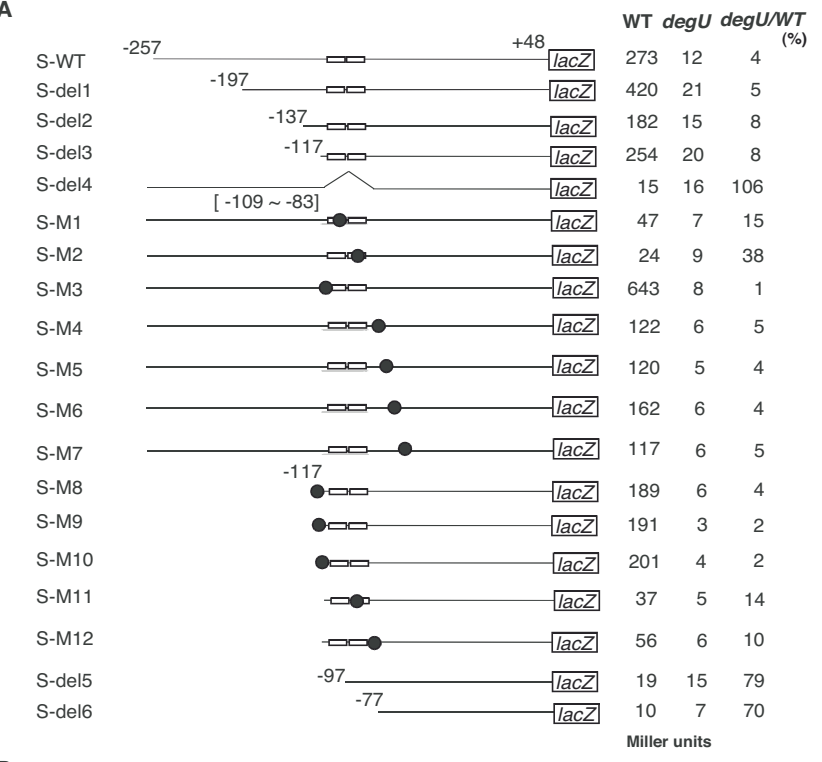

B

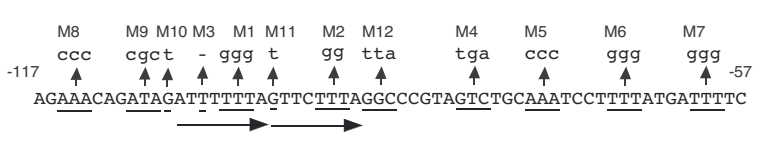

\section{Figure 6}

Expression of the various sacB-lacZ fusions. A. The $5^{\prime}$ and $3^{\prime}$ endpoints of sacB-lac $Z$ are indicated. The open rectangles on the line indicate the DegUbinding motifs. The mutant fusions tested included the del series of fusions, in which increasingly large deletions were made from the $5^{\prime}$ end, and the $M$ series, in which point mutations (replaced nucleotides are shown in $B . M 3$ is the mutation where one T residue is deleted.) were introduced (indicated by the filled circles on the line). All fusions were constructed by cloning various PCR products into pIS284. Consequently, all strains are derivatives of OAM37 I (amyE::sacB-lacZ). The oligonucleotides used to construct the pIS284 derivatives are shown in Additional file I. The $\beta$-galactosidase activities of sacB-lac $Z$ were measured from cells grown in $\mathrm{MC}$ medium containing I M NaCl as described previously [45]. The activities were assayed hourly from T0 through to T4. At least three independent assays were performed and the averages of the peak values are shown. Standard deviations did not exceed I5\%. The "degU" columns show the $\beta$-galactosidase activities of each fusion in the $\operatorname{degU}:: \mathrm{Km}^{\mathrm{r}}$ background.

\section{Changing the $D R$ to an $I R$ in the sacB promoter results in degS-independent but degU-dependent regulation}

According to the above results, it is possible that the IRlike and the DR sequences positively regulate gene expression by unphosphorylated DegU and DegU-P, respectively. The expression of $a p r E$ and $s a c B$, whose promoters carry a DR, is positively regulated by DegU-P (Figure 4 and Figure 6) [19]. In addition, we found recently that three DRs with zero or two-nucleotide spacing serve as positive cis-elements for DegU-P in the regulatory region of $b p r$, which is a DegU-P-regulated gene [22]. In contrast, the expression of $\operatorname{comK}$ and $f l g B$, whose promoters carry IR and IR-like sequences, respectively, is regulated by unphosphorylated DegU (Figure 1 and Figure 3) [19]. Thus, we examined the effect of changing a DR to an IR on 
degS-dependent expression of the sacB-lacZ fusion by replacing the downstream half of the $s a c B$ DR by that of $\operatorname{comK}$ IR with various spacers. The expression of wild-type $s a c B-l a c Z$ was dependent on both the $\operatorname{deg} S$ and $\operatorname{deg} U$ genes (Figure 7A). In the case of SC-IR2, expression of the fusion was not affected when the degS mutation was introduced, although the expression was still dependent on functional $\operatorname{deg} U$ (Figure 7B). This strongly suggested that changing the DR to an IR results in the appearance of unphosphorylated DegU-dependent positive regulation. The other series of SC-IR fusions showed similar regulation patterns to that of SC-IR2 (Figure 7C). Next, we reversed the orientation of the downstream half site of $s a c B$ DR to make an artificial IR in the $s a c B$ promoter, which had the original sequence except for the insertion of one $\mathrm{G}$ nucleotide to make two-base spacing. This change rendered the expression of the SC-IR fusion resistant to the degS mutation to some extent. Furthermore, unphosphoylated DegU was able to bind to the IR in SC-IR4 in the footprint analysis (Figure 8). These results are further supports of our hypothesis.

A

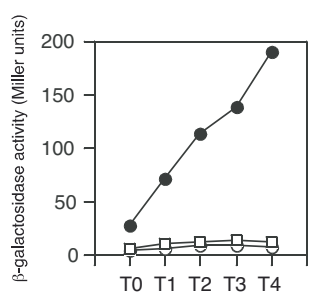

C

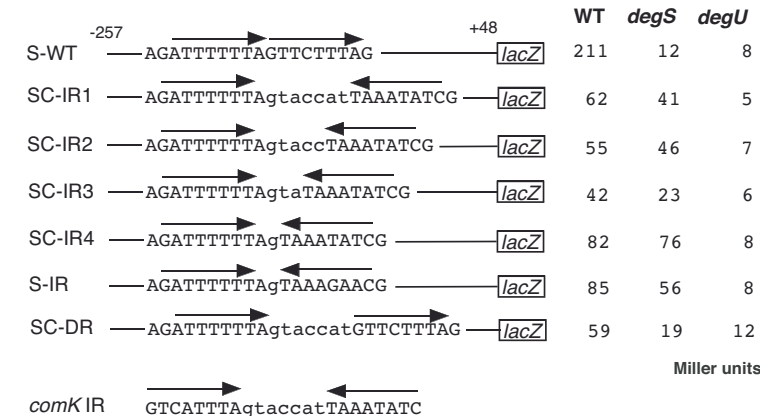

Figure 7

Effect of arrangement of the DegU-recognition motifs in the sacB promoter. All fusions constructed by cloning various PCR products into pIS284 are derivatives of the S-WT fusion and the oligonucleotides used to construct are shown in Additional file I. The $\beta$-galactosidase activities of sacB-lac $Z$ were measured from cells grown in $M C$ medium containing I $M$ $\mathrm{NaCl}$ as described previously [45]. A and B. Filled circles, degS $S^{+} \operatorname{deg} U^{+}$; open circles, degS; squares, degU. The S-WT (A) and SC-IR2 (B) fusions are shown. $C$. The activities were assayed hourly from T0 through to T4. At least three independent assays were performed and the averages of the peak values are shown. Standard deviations did not exceed $10 \%$. The "degS" and "degU" columns show the $\beta$-galactosidase activities of each fusion in the degS::Em ${ }^{r}$ and degU::Km ${ }^{r}$ backgrounds, respectively. Arrows above the sequences indicate the DegU-binding motifs.

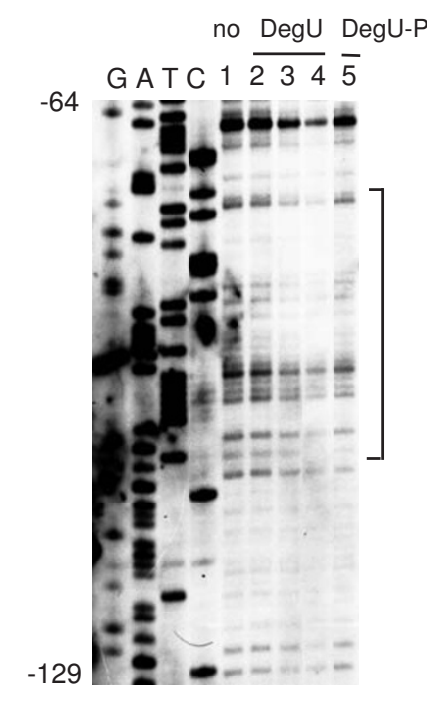

$-113 \longrightarrow \longrightarrow 44$ CAGATAGATTTTTTAGTAAATATCGGCCCGTAGTCTGCAA GTCTATСTAАAААATCATTTATAGCCGGGCATCAGACGTT

\section{Figure 8}

Footprint analysis of the $s a c B$ promoter carrying IR. To analyze the top strand, the oligonucleotide pair sacB-bio3 and sacB-D was used for PCR amplification from the plasmid carrying the SC-IR4 fusion (figure 7). The procedures for sequencing ladder generation and footprint analysis were the same as those in figure 5 . The probe $(40 \mathrm{nM})$ was incubated with increasing amounts of DegU and His-DegS in the presence or absence of ATP, and subjected to DNase I cleavage. The sequencing ladder is shown in lanes G, A, T and C. Lane I. No protein; 2, $0.15 \mu \mathrm{M}$ of DegU and 0.05 $\mu \mathrm{M}$ His-tagged DegS; 3, $0.3 \mu \mathrm{M}$ of DegU and 0.1 $\mu \mathrm{M}$ His-tagged DegS; 4 and 5; $0.6 \mu \mathrm{M}$ of DegU and $0.2 \mu \mathrm{M}$ His-tagged DegS. 2, 3 and 4, no ATP; 5, I mM ATP. The nucleotide sequence of the promoter region is shown. The bracket indicates the protected region. Arrows above the sequences and the numbers indicate the DegU-binding motifs and the nucleotide positions relative to the transcription start site, respectively.

Another difference between the promoters of DegU-regulated and DegU-P-regulated genes is that the IR of $\operatorname{comK}$ and IR-like sequences of $f \lg B$ (BR1 and BR2) have long spacing regions, whereas the DRs of aprE, bpr and $s a c B$ have short spacing regions. Thus, we examined whether a long spacer between the two half sites of $s a c B$ DR might alter its regulation. The introduction of a long spacer corresponding to that of the comK IR into the DR of $s a c B$ (SC$\mathrm{DR})$ did not affect $d e g S$-dependent positive regulation of $s a c B$ (Figure 7C). This suggests that spacing between two half-sites would not be a critical factor for the promoter selectivity of DegU.

\section{Discussion}

This study demonstrated that while phosphorylation of DegU stimulates its binding to the DR in the $s a c B$ promoter, it inhibits DegU-binding to the artificial IR of the same promoter. These results strongly suggested that DegU-P prefers binding to DR but not to IR (Figure 9A). This hypothesis is consistent with the observation that 
phosphorylation of DegU abolished its binding to BR1 containing the IR-like sequence in the flgB promoter under the condition used. DegU-P, however, could bind to the BR2 in $f \lg B$ and the degU32 mutation resulted in positive regulation of the F1 fusion through BR1, suggesting that DegU-P could bind to this region when cellular concentrations of DegU-P were high enough. We note that this positive regulation of the degU32 mutation was observed only in the swrAA background. The possible binding of DegU-P to the flgB promoter may be an exception to the hypothesis and remains to be elucidated.

Notably, DegU belongs to the NarL response regulator family, which is characterized by a classical helix-turnhelix domain that recognizes IRs of the same motifs [30]. However, NarL has also been reported to bind to differently arranged motifs, namely, DRs [31]. Indeed, another report has shown that the NarL-binding sites in various promoters are differently arranged as direct repeats, monomers, or inverted or divergent repeat [32]. It is quite possible that the binding to differently arranged motifs requires different protein-protein interactions within the putative dimers of DegU, which might be regulated by phosphorylation. Global analysis of the DegU regulon has identified many genes other than the genes analyzed in this study $[8,9]$. It would be of interest to determine how the DegU motif is arranged in the promoters of these genes as this may further enhance our understanding of DegU regulation.

On the basis of the DegU-binding sites we have identified, we propose that 5'-GNCATTTA-3' is the consensus DNAbinding sequence of DegU and DegU-P (Figure 9B). Supporting this is a previous study, which also proposed a putative DegU consensus sequence on the basis of genetic analysis of the wapA promoter [7]: our newly proposed consensus is consistent with the important sequences for DegU-binding to the wapA promoter. The DegU-binding sequences that we identified are rather degenerate, and we speculate that this may be a common feature of the sequences recognized by other global regulators. Therefore, it may be difficult to identify DegU-binding sequences by inspecting of the upstream regions of DegUregulon genes. For example, there is DegU-consensus-like sequences in the $s a c B$ promoter region (-72 to -57$)$, and the region appeared to be protected from DNase I cleavage to some extent. The mutational analysis, however, revealed that the sequences do not serve as a functional cis-element for DegU-P (S-M6 and S-M7 in Figure 6). Unknown features other than the primary sequence of the DNA target may be involved in DegU-P regulation.

We observed that His-tagged unphosphorylated DegU bound to the promoter regions of $a p r E$ and sacB (Figure 5) $[14,19]$. This might mean that His-tagged unphosphor-
A

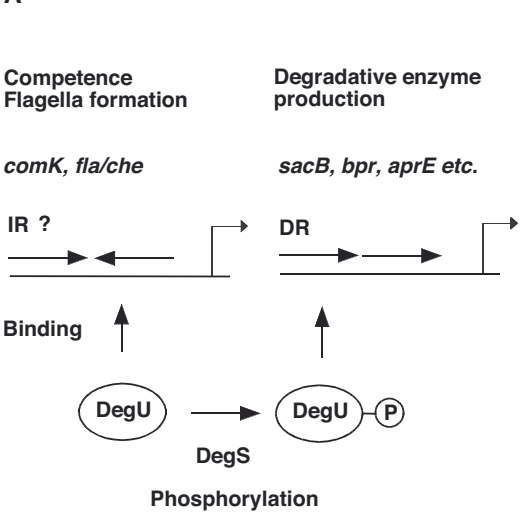

B

\section{Figure 9}

Schematic representation of the regulation of the DegU-regulon. A. Unphosphorylated DegU binds to the BRs containing the IR-like sequences in the flgB promoter or IR in the comK promoter. DegS phosphorylates DegU in response to some unknown signal. DegU-P binds to DR in the $s a c B$, as well as probably to the $b p r$ and $a p r E$ promoters. B. Alignment of DegU-recognition sequences. comK-IR and aprE-DR are shown in [19] and bpr-DRs are shown in [22].

ylated DegU can bind to DR. This characteristic is different from that observed for intact unphosphorylated DegU. It was reported that His-tagged Spo0A was phosphorylated in E. coli, resulting in an isolation of Spo0A-P without any phosphorylation reaction [33]. Thus, it is possible that His-tagged DegU purified from E. coli might undergo phosphorylation and serve as DegU-P. The possibility is unlikely, however, because a half-life of DegU-P has been reported to be less than 90 min due to an intrinsic phosphatase activity of DegU [5]. It has been shown that the addition of the His-tag to the C-terminus of Salmonella PhoP response regulator affects its biochemical properties and conformation with respect to its dimer formation and DNA-binding ability [34]. Addition of His-tag at the Nterminus of DegU might alter its conformation as in the case of Salmonella PhoP.

In our analysis of the mechanisms regulating $f l g B$ expression, we observed that SwrAA requires DegU-binding to $B R 1$ to enhance $f l g B$ expression. While the exact function of SwrAA is not yet understood, it is known that swrAA is required for swarming motility $[26,35], \gamma$-poly-glutamic acid synthesis [36] and enhancing of the transcription of the large fla/che operon in a non-laboratory strain [25]. The result that SwrAA stimulates the fla/che transcription in a DegU-dependent manner led us to a speculation that SwrAA might facilitate DegU-binding to its target promoter or modulate the DegU function. We noted that if SwrAA functions to enhance the expression of some DegU-dependent genes, it remains to be determined how SwrAA focuses specifically on its targets among the many genes that are regulated by DegU. 
Amati et al. showed that the downstream region from the flgB transcription start site containing BR2 did not interact with DegU [24]. This result is inconsistent with our footprint data (Figure 1 and Figure 2). We do not know the reason for this discrepancy. In addition, they claimed that DegU-binding to the upstream region resulted in repression of $y l x F$, which is the ninth gene of the fla/che operon. Our result clearly showed that the expression of the F1 fusion containing only BR1 was increased in degU32 cells (Figure 3B). The different interpretation between their results and ours might be due to the use of $y l x F-l a c Z$ in their analysis. DegU-P bound to BR1 could be somehow dysfunctional in the presence of $\mathrm{BR} 2$. The regulatory mechanism involving the two BRs remains to be solved, although we speculate that DegU-P bound at each region might interact together through DNA-looping, leading to the abolishment of the positive effect of DegU-P through $\mathrm{BR} 1$. As a result, in the regulatory region carrying both regions, the degU32 mutation results in a decrease in $f \lg B$ expression. This could be caused by a decrease in the cellular concentrations of unphosphorylated DegU, but not in the repressor function of DegU-P at BR2, as BR2 did not serve as a functional cis-acting site in the absence of BR1. DegU-P at BR2 might rather work as an anti-activator.

We found that unphosphorylated DegU is a positive regulatory factor of the $f \lg B$ promoter, which is the first gene in the fla/che operon (including the sigD gene that encodes sigma D factor). It was reported that disruption of degU decreases the expression of sigD [37], which is governed by the upstream $f l g B$ promoter $[23,38,39]$. Our findings are consistent with this observation.

Recently it has been reported that swarming motility and the $f \lg B$ expression in the non-laboratory strain requires DegS-independent low-level of DegU-P [40,41]. These observations may be consistent with our findings, since unphosphorylated DegU (or DegU phosphorylated at a low level) may positively regulate the expression of the fla/ che operon. To further characterize the DegS-independent phosphorylation of DegU, a study using a DegU mutant lacking the phosphorylation site should be needed.

\section{Conclusion}

This study demonstrated that DegU positively regulates $f \lg B$ and $s a c B$ via the BRs containing the IR-like sequences and the DR present in their promoter regions, respectively (Figure 9). In, addition, we showed that DegU-P prefers binding to DR but not to IR in the regulatory region of the $s a c B$ promoter.

\section{Methods}

\section{Bacterial strains and culture media}

All the B. subtilis strains used for this study are listed in Table 1. One-step competence medium (MC) was used
[6]. For DNA manipulation, Escherichia coli cells were grown in LB medium. The concentrations of antibiotics used have been described previously [42].

\section{Plasmid construction}

Synthetic oligonucleotides were commercially prepared by the Tsukuba Oligo Service (Ibaraki, Japan). The plasmids and oligonucleotides used in this study are listed in Table 1 and Additional file 1, respectively. We used total DNA from B. subtilis 168 as the PCR template unless otherwise indicated. To construct pDG-swrAN, a PCR product produced by using swrA-FA and swrA-FB and total DNA from B. subtilis IFO3335 was digested by BamHI and EcoRI and cloned into PDG1731 treated with the same restriction enzymes [43]. To construct pTYB-degU, a PCR product produced by degU-Chi-N and degU-Chi-S was digested by NdeI and SmaI and cloned into pTYB2 treated with the same restriction enzymes. To construct pCa-yvyE, a PCR product produced by using yvyE-F1 and yvyE-R1 was digested by HindIII and BamHI and cloned into pCA191 treated with the same restriction enzymes [44]. To construct pIS284-flgB and pIS284-sacB, PCR products were prepared by using the primer pairs, flgB-F2 and flgBDD, and sacB-U and sacB-D, respectively. The DNA fragments were digested with BamHI and HindIII and ligated into the similarly-digested pIS284 plasmid. The resultant plasmids were transformed into 168 after linearization by Pst I digestion, thereby generating the strain carrying F1D and S-WT, respectively. The del series of sacB-lacZ fusions, the F1 to F3 derivatives, and F2D were constructed by generating PCR products using oligonucleotides corresponding to different 5'-termini or 3' termini of the fusions and cloning these fragments into pIS284. The $M$ series of flgBlacZ and $s a c B$-lacZ fusions and the SC series of $s a c B-l a c Z$ fusions as well as S-IR were constructed by cloning mutagenized PCR products into pIS284. Site-directed mutagenesis was performed by an oligonucleotide-based PCR method as described previously [45]. S-del4 was constructed by site-directed mutagenesis using oligonucleotides sacB-del-1, sacB-del-8, sacB-U and sacB-D. S-M3 was obtained by PCR error during the construction of $\mathrm{S}$ WT. The resulting fusion-bearing plasmids were used to transform 168. The sequences cloned into all plasmids were confirmed.

\section{Purification of soluble His-tagged DegS, His-tagged DegU and intact DegU proteins}

The recombinant His-tagged DegS, His-tagged DegU and chitin-binding domain (CBD)- and intein-fused DegU proteins were induced in E. coli BL21 carrying pET-degS, M15 carrying pRep4 and pDG-His-degU, and BL21 carrying pTYB-degU by previously described methods [14]. His-tagged DegS was produced as insoluble protein and its pellets were resuspended in $2.5 \mathrm{ml}$ of buffer A [ $6 \mathrm{M}$ guanidine- $\mathrm{HCl}, 150 \mathrm{mM} \mathrm{NaCl}$, and $50 \mathrm{mM}$ Tris- $\mathrm{HCl}(\mathrm{pH}$ 
Table I: Strains and plasmids used for this study.

\begin{tabular}{|c|c|c|}
\hline Strain & Genotype & Reference or source \\
\hline 168 & $\operatorname{trpC2}$ & Laboratory stock \\
\hline IFO3335 & Non-laboratory strain & Laboratory stock \\
\hline IA95 & $\operatorname{trp} C 2 \operatorname{deg} U 32$ & BGSC \\
\hline OAM392 & $\operatorname{trp} C 2$ thrC ::swrAA N (Spr) & This study \\
\hline TT7।4 & $\operatorname{trpC2}$ leuC7 $\operatorname{deg} U\left(\mathrm{Km}^{\mathrm{r}}\right)$ & [46] \\
\hline TT7।9 & $\operatorname{trp} C 2$ leuC7 $\Delta$ degS (in frame deletion) & [46] \\
\hline OAM369 & 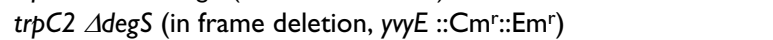 & This study \\
\hline OAM385 & $\operatorname{trpC2}$ amyE ::flgB-lacZ ID $\left(\mathrm{Cm}^{r}\right)$ & This study \\
\hline OAM386 & $\operatorname{trp} C 2$ amyE ::flgB-lacZ ID $\left(\mathrm{Cm}^{r}\right)$ thrC ::swrAA N (Spr) & This study \\
\hline OAM387 & $\operatorname{trpC2}$ amyE ::flgB-lacZ ID $\left(\mathrm{Cm}^{\mathrm{r}}\right) \operatorname{deg} U\left(\mathrm{Km}^{\mathrm{r}}\right)$ & This study \\
\hline OAM388 & $\operatorname{trpC2}$ amyE ::flgB-lacZ ID $\left(\mathrm{Cm}^{r}\right) \Delta \operatorname{degS}\left(\mathrm{Em}^{r}\right)$ & This study \\
\hline OAM389 & $\operatorname{trpC2}$ amyE ::flgB-lacZ ID $\left(\mathrm{Cm}^{r}\right)$ thrC ::swrAA N (Spr) degU $\left(\mathrm{Km}^{\mathrm{r}}\right)$ & This study \\
\hline OAM390 & $\operatorname{trpC2}$ amyE ::flgB-lacZ ID $\left(\mathrm{Cm}^{r}\right)$ thrC ::swrAA N $\left(\mathrm{Sp}^{r}\right) \Delta \operatorname{deg} S\left(\mathrm{Em}^{\mathrm{r}}\right)$ & This study \\
\hline OAM39I & $\operatorname{trp} C 2$ amyE ::flgB-lacZ ID $\left(\mathrm{Cm}^{r}\right) \operatorname{degU} 32$ & This study \\
\hline OAM37I & $\operatorname{trpC2}$ amyE ::sacB-lacZ S-WT $\left(\mathrm{Cm}^{\mathrm{r}}\right)$ & This study \\
\hline OAM372 & $\operatorname{trpC2}$ amyE ::sacB-lacZ S-WT $\left(\mathrm{Cm}^{\mathrm{r}}\right) \operatorname{deg} U\left(\mathrm{Km}^{\mathrm{r}}\right)$ & This study \\
\hline OAM373 & $\operatorname{trpC2}$ amyE ::sacB-lacZ S-WT $\left(\mathrm{Cm}^{\mathrm{r}}\right) \Delta \operatorname{degS}\left(\mathrm{Em}^{\mathrm{r}}\right)$ & This study \\
\hline OAM393 & $\operatorname{trp} C 2$ amyE ::sacB-lacZ SC-IR2 $\left(\mathrm{Cm}^{r}\right)$ & This study \\
\hline OAM394 & $\operatorname{trpC2}$ amyE ::sacB-lacZ SC-IR2 $\left(\mathrm{Cm}^{\mathrm{r}}\right) \operatorname{degU}\left(\mathrm{Km}^{\mathrm{r}}\right)$ & This study \\
\hline OAM395 & $\operatorname{trpC2}$ amyE ::sacB-lacZ SC-IR2 $\left(\mathrm{Cm}^{r}\right) \Delta \operatorname{degS}\left(\mathrm{Em}^{\mathrm{r}}\right)$ & This study \\
\hline Plasmid & Description & Reference or source \\
\hline pDG-swrAAN & pDGI73I carrying swrAA N & This study \\
\hline PCa-yvyE & $\mathrm{PCA} / 9 \mathrm{I}$ carrying a part of $y v y E$ and a promoter of degSU & K. Kobayashi \\
\hline $\mathrm{pCm}:: \mathrm{Em}^{\mathrm{r}}$ & Ampicillin and erythromycin resistance & [48] \\
\hline PTYB-degU & PTYB2 carrying degU, ampicillin resistance & This study \\
\hline pET-degS & pET28b carrying degS, kanamycin resistance & K. Kobayashi \\
\hline pDG-His-degU & Plasmid carrying T5 promoter-His6-degU, ampicillin resistance & [19] \\
\hline pIS284 & Insertion vector to amyE, chloramphenicol resistance, lacZ & I. Smith \\
\hline pIS284-flgB & pIS284 carrying a promoter region of flgB & This study \\
\hline pIS284-sacB & pIS284 carrying a promoter region of $s a c B$ & This study \\
\hline
\end{tabular}

7.6)] and left for $1 \mathrm{hr}$ at room temperature. Two $\mathrm{ml}$ of $50 \%$ Ni-NTA resin (Qiagen) equilibrated by buffer A were added to the sample, which was then shaken gently for 45 min at room temperature. The resultant suspension (about $5 \mathrm{ml}$ ) was packed in a mini-column, washed first with $10 \mu \mathrm{l}$ of buffer A and then with $5 \mathrm{ml}$ of buffer B [150 $\mathrm{mM} \mathrm{NaCl}$ and $50 \mathrm{mM}$ Tris-HCl (pH 7.6)] containing $8 \mathrm{M}$ urea. Renaturation of His-tagged DegS was performed by removing urea, which was done in a step-wise fashion by passing $5 \mathrm{ml}$ each of buffer B containing $6 \mathrm{M}, 4 \mathrm{M}$ and 2 $\mathrm{M}$ urea through the column. Finally, the column was washed with $5 \mathrm{ml}$ of buffer B containing $10 \mathrm{mM}$ imidazole. The protein was then eluted with buffer B containing $0.5 \mathrm{M}$ imidazole. His-tagged DegU was produced as a soluble protein in E. coli and purification was performed by step-wise elution from a Ni-affinity column with imidazole as described previously [14]. CBD- and intein-fused DegU was purified by using chitin-coupled resin and autoactivating intein by DTT according to the manufacturer's recommendations (New England Biolab inc). After SDS-PAGE analysis of the fractions, the purified proteins were dialyzed against TEDG buffer [46]. Aliquots of the purified proteins were stored at $-80^{\circ} \mathrm{C}$.

\section{Footprint assays using DegU-P}

Phosphorylation of DegU and the binding of DegU-P to the probe DNA were carried out by incubating intact DegU and His-tagged DegS at $25^{\circ} \mathrm{C}$ for $30 \mathrm{~min}$ (molar ratio $3: 1$ ) with a biotinylated DNA probe, i.e., in a buffer containing protein solution (TEDG buffer). The final concentration of the reaction mixture $(65 \mu \mathrm{l})$ for the footprint analysis was as follows; $10 \mathrm{mM}$ (NH4)2SO4, $1 \mathrm{mM}$ DTT, $0.2 \%$ Tween 20, $5 \mathrm{mM} \mathrm{MgCl2,} 31 \mathrm{mM}$ Tris- $\mathrm{HCl}$ ( $\mathrm{pH} 7.5)$, $0.3 \mathrm{mM}$ EDTA, $44 \mathrm{mM} \mathrm{KCl}, 1 \mu \mathrm{g}$ poly dI-dC and $1 \mathrm{mM}$ ATP. Immediately at the end of the reaction, 4 units of DNase I (Roche, Indianapolis, USA) in $7.5 \mu \mathrm{l}$ buffer containing 6\% glycerol, $10 \mathrm{mM}$ Tris- $\mathrm{HCl}(\mathrm{pH} 7.5), 50 \mathrm{mM}$ $\mathrm{NaCl}, 5 \mathrm{mM} \mathrm{MgCl}$, $1 \mathrm{mM}$ DTT and $1 \mathrm{mg}$ bovine serum albumin was added. The reaction mixture was left at room temperature for $5 \mathrm{~min}$ and then subjected to phenol extraction and subsequent ethanol precipitation after the addition of stop solution (0.1\% SDS, 20 mM EDTA, 200 
$\mathrm{mM} \mathrm{NaCl}, 40 \mu \mathrm{g} / \mathrm{ml}$ tRNA). After the addition of a loading dye, the samples were applied onto a $6 \%$ polyacrylamide gel. The probes were prepared by PCR using the oligonucleotide pairs indicated in Additional file 1. Detection of biotinylated DNA and preparation of sequencing ladder were done by using the methods described previously [14].

\section{Authors' contributions}

KT carried out protein purification and footprint analysis. MO carried out construction of plasmids and strains, and in vivo lac $Z$ analysis and wrote the paper. All the authors have read and approved the final manuscript.

\section{Additional material}

\section{Additional file 1}

Oligonucleotides used for this study. Oligonucleotide sequences used for this study.

Click here for file

[http://www.biomedcentral.com/content/supplementary/1471-

2180-8-8-S1.xls]

\section{Acknowledgements}

We thank K. Kobayashi for communicating unpublished results and the kind gift of plasmids. We also thank P. Stragier and I. Smith for the kind gift of plasmids. We are indebted to K. Shimane and T. Ohsawa for technical assistance. This work was supported by a Grant-in-aid for Scientific Research (C) from the Ministry of Education, Culture, Sports, Science, and Technology of Japan and research grants from KANPOU Science Foundation and Takano Agricultural Chemistry Foundation.

\section{References}

I. Stock AM, Robinson VL, Goudreau PN: Two-component signal transduction. Annu Rev Biochem 2000, 69:183-215.

2. Lewis RJ, Scott DJ, Brannigan JA, Ladds JC, Cervin MA, Spiegelman GB, Hoggett JG, Barak I, Wilkinson AJ: Dimer formation and transcription activation in the sporulation response regulator Spo0A. J Mol Biol 2002, 3 I 6:235-245.

3. Da Re S, Bertagnoli S, Fourment J, Reyrat JM, Kahn D: Intramolecular signal transduction within the FixJ transcriptional activator: in vitro evidence for the inhibitory effect of the phosphorylatable regulatory domain. Nucleic Acids Res 1994, 22:|555-156|.

4. Fabret C, Feher VA, Hoch JA: Two-component signal transduction in Bacillus subtilis: how one organisim sees its world. J Bacteriol 1999, I81:1975-1983.

5. Dahl MK, Msadek T, Kunst F, Rapoport G: The phosphorylation state of the DegU response regulator acts as a molecular switch allowing either degradative enzyme synthesis or expression of genetic competence in Bacillus subtilis. J Biol Chem 1992, 267:|4509-|45|4.

6. Kunst F, Msadek T, Rapoport G: Signal transduction network controlling degradative enzyme synthesis and competence in Bacillus subtilis. In Regulation of Bacterial Differentiation Edited by: Piggot PJ, Moran CP Jr, Youngman P. Washington, DC: ASM Press; 1994: I-20.

7. Dartois V, Debarbouille M, Kunst F, Rapoport G: Characterization of a novel member of the DegS-DegU regulon affected by salt stress in Bacillus subtilis. J Bacteriol 1998, I 80: I855-I86I.

8. Ogura M, Yamaguchi H, Yoshida K, Fujita Y, Tanaka T: DNA microarray analysis of Bacillus subtilis DegU, ComA and PhoP reg- ulons: an approach to comprehensive analysis of $B$. subtilis two-component regulatory systems. Nucleic Acids Res 2001, 29:3804-3813.

9. Mader U, Antelmann H, Buder T, Dahl MK, Hecker M, Homuth G: Bacillus subtilis functional genomics: genome-wide analysis of the DegS-DegU regulon by transcriptomics and proteomics. Mol Genet Genomics 2002, 268:455-467.

10. Kunst F, Rapoport G: Salt stress is an environmental signal affecting degradative enzyme synthesis in Bacillus subtilis. J Bacteriol 1995, I 77:2403-3407.

II. Steil L, Hoffmann T, Budde I, Volker U, Bremer E: Genome-wide transcriptional profiling analysis of adaptation of Bacillus subtilis to high salinity. J Bacteriol 2003, I 85:6358-6370.

12. Dervyn E, Noirot-Gros MF, Mervelet P, McGovern S, Ehrlich SD, Polard P, Noirot P: The bacterial condensin/cohesin-like protein complex acts in DNA repair and regulation of gene expression. Mol Microbiol 2004, 5 I: 1629-1640.

13. Pottathil M, Lazazzera B: The extracellular Phr peptide-Rap phosphatase signaling circuit of Bacillus subtilis. Front BioSci 2003, 8:32-45.

14. Ogura M, Shimane K, Asai K, Ogasawara N, Tanaka T: Binding of response regulator DegU to the aprE promoter is inhibited by RapG, which is counteracted by extracellular PhrG in Bacillus subtilis. Mol Microbiol 2003, 49: I685-1697.

15. Hayashi K, Kensuke T, Kobayashi K, Ogasawara N, Ogura M: Bacillus subtilis RghR (YvaN) represses rapG and rapH, which encode inhibitors of expression of the srfA operon. Mol Microbiol 2006, 59:1714-1729.

16. Grossman AD: Genetic networks controlling the initiation of sporulation and the development of genetic competence in Bacillus subtilis. Annu Rev Genetics 1995, 29:477-508.

17. Dubnau $D$, Lovett $C M$ Jr: Transformation and recombination. In Bacillus subtilis and its closest relatives: from genes to cells Edited by: Sonenshine AL, Hoch JA, Losick R. Washington, DC: ASM Press; 2002:453-472

18. Hamoen LW, Van Werkhoven AF, Venema G, Dubnau D: The pleiotropic response regulator DegU functions as a priming protein in competence development in Bacillus subtilis. Proc Natl Acad Sci USA 2000, 97:9246-925I.

19. Shimane K, Ogura M: Mutational analysis of the helix-turn-helix region of Bacillus subtilis response regulator DegU, and identification of cis-acting sequences for DegU in the aprE and comK promoters. J Biochem 2004, 136:387-397.

20. Ferrari E, Henner DJ, Perego M, Hoch JA: Transcription of Bacillus subtilis subtilisin and expression of subtilisin in sporulation mutants. J Bacteriol 1988, I 70:289-295.

21. Sloma A, Rufo GA Jr, Rudolph CF, Sullivan BJ, Theriault KA, Pero J: Bacillopeptidase $F$ of Bacillus subtilis: purification of the protein and cloning of the gene. J Bacteriol 1990, I 72: |470-|477.

22. Tsukahara K, Ogura M: Characterization of DegU-dependent expression of bpr in Bacillus subtilis. FEMS Microbiol Lett in press.

23. Estacio W, Anna-Arriola SS, Adedipe M, Marquez-Magana LM: Dual promoters are responsible for transcription initiation of the fla/che operon in Bacillus subtilis. J Bacteriol 1998, 180:3548-3555.

24. Amati G, Bisicchia P, Galizzi A: DegU-P represses expression of the motility fla-che operon in Bacillus subtilis. J Bacteriol 2004, I 86:6003-60|4.

25. Kearns DB, Losick R: Cell population heterogeneity during growth of Bacillus subtilis. Genes Dev 2005, 19:3083-3094.

26. Kearns DB, Chu F, Rudner R, Losick R: Genes governing swarming in Bacillus subtilis and evidence for $a$ phase variation mechanism controlling surface motility. Mol Microbiol 2004, 52:357-369.

27. Klier A, Fouet A, Debarbouille M, Kunst F, Rapoport G: Distinct control sites located upstream from the levansucrase gene of Bacillus subtilis. Mol Microbiol 1987, I:233-24I.

28. Shimotsu $H$, Henner DJ: Modulation of Bacillus subtilis levansucrase gene expression by sucrose and regulation of the steady-state mRNA level by sacU and sacQ genes. I Bacteriol 1986, 168:380-388.

29. Henner DJ, Ferrari E, Perego M, Hoch JA: Location of the targets of the hpr-97, sacU32(Hy), and sacQ36(Hy) mutations in upstream regions of the subtilisin promoter. J Bacteriol 1988 , I 70:296-300.

30. Harrison SC, Aggarwal AK: DNA recognition by proteins with the helix-turn-helix motif. Annu Rev Biochem 1990, 59:933-969. 
31. Maris AE, Sawaya MR, Kaczor-Grzeskowiak M, Jarvis MR, Bearson SM, Kopka ML, Schroder I, Gunsalus RP, Dickerson RE: Dimerization allows DNA target site recognition by the NarL response regulator. Nat Struct Biol 2002, 9:77I-778.

32. Darwin AJ, Tyson KL, Busby SJ, Stewart V: Differential regulation by the homologous response regulators NarL and NarP of Escherichia coli K-I 2 depends on DNA binding site arrangement. Mol Microbiol 1997, 25:583-595.

33. Ladds CJ, Muchova K, Blaskovic D, Lewis RJ, Brannigan JA, Wilkinson A], Barak I: The response regulator Spo0A from Bacillus subtilis is efficiently phosphorylated in Escherichia coli. FEMS Microbiol Lett 2003, 223: 153-157.

34. Perron-Savard P, De Crescenzo G, Le Moual H: Dimerization and DNA binding of the Salmonella enterica PhoP response regulator are phosphorylation independent. Microbiology 2005, | 5 |:3979-3987.

35. Calvio C, Celandroni F, Ghelardi E, Amati G, Salvetti S, Ceciliani F, Galizzi A, Senesi S: Swarming differentiation and swimming motility in Bacillus subtilis are controlled by swrA, a newly identified dicistronic operon. J Bacteriol 2005, | 87:5356-5366.

36. Stanley NR, Lazazzera BA: Defining the genetic differences between wild and domestic strains of Bacillus subtilis that affect poly-gamma-dl-glutamic acid production and biofilm formation. Mol Microbiol 2005, 57: I I43-I I 58.

37. Tokunaga T, Rashid MH, Kuroda A, Sekiguchi J: Effect of degS-degU mutations on the expression of sigD, encoding an alternative sigma factor, and autolysin operon of Bacillus subtilis. J Bacteriol I994, I 76:5I77-5I80.

38. Zuberi AR, Ying CW, Weinreich MR, Ordal GW: Transcriptional organization of a cloned chemotaxis locus of Bacillus subtilis. J Bacteriol 1990, I 72: 1870-1876.

39. Marquez-Magana LM, Chamberlin MJ: Characterization of the sigD transcription unit of Bacillus subtilis. J Bacteriol 1994, I 76:2427-2434.

40. Verhamme DT, Kiley TB, Stanley-Wall NR: DegU co-ordinates multicellular behavior exhibited by Bacillus subtilis. Mol Microbiol 2007, 65:554-568.

4I. Kobayashi K: Gradual activation of the response regulator DegU controls serial expression of genes for flagellum for mation and biofilm formation in Bacillus subtilis. Mol Microbiol 2007, 66:395-409.

42. Ogura $M$, Ohshiro $\mathrm{Y}$, Hirao S, Tanaka T: A new Bacillus subtilis gene, med, encodes a positive regulator of comK. J Bacteriol 1997, I 79:6244-6253.

43. Guerout-Fleury AM, Frandsen N, Stragier P: Plasmids for ectopic integration in Bacillus subtilis. Gene 1996, I 80:57-6I.

44. Murakami T, Haga K, Takeuchi M, Sato T: Analysis of the Bacillus subtilis spolIIJ Gene and Its Paralogue Gene, yqjG. J Bacteriol 2002, I 84: I998-2004.

45. Ogura M, Tanaka T: Transcription of Bacillus subtilis degR is sig$\mathrm{ma}^{\mathrm{D}}$-dependent and suppressed by multicopy proB through sigmaD. J Bacteriol 1996, I 78:216-222.

46. Mukai K, Kawata M, Tanaka T: Isolation and phosphorylation of the Bacillus subtilis degS and degU gene products. J Biol Chem 1990, 265:20000-20006.

47. Hayashi K, Ohsawa T, Kobayashi K, Ogasawara N, Ogura M: The H2O2 stress-responsive regulator PerR positively regulates srfA expression in Bacillus subtilis. J Bacteriol 2005 , | 87:6659-6667.

48. Steinmetz M, Richter R: Plasmids designed to alter the antibiotic resistance expressed by insertion mutations in Bacillus subtilis, through in vivo recombination. Gene 1994, I42:79-83.

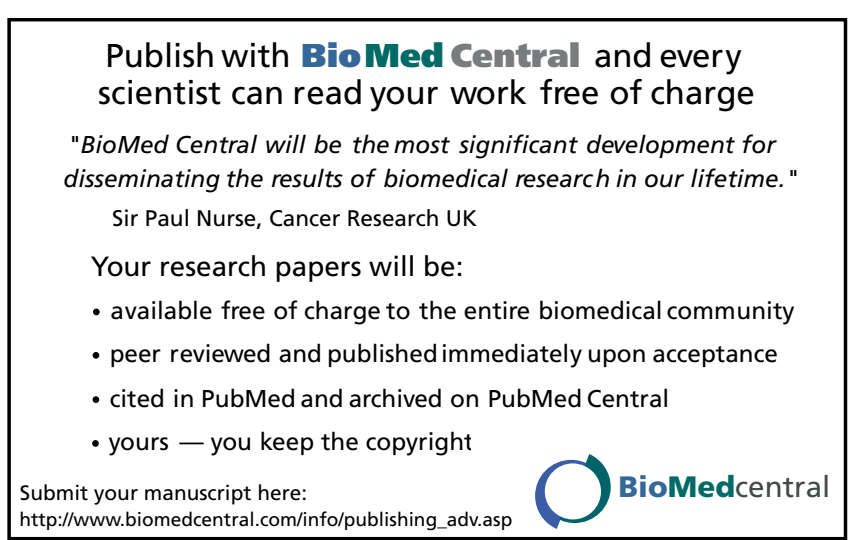

